\title{
GAS-CHROMATOGRAPHIC FRACTIONATIONS OF STABLE ISOTOPES OF \\ CARBON AND OXYGEN IN CARBON DIOXIDE
}

by Allan T. Shepard ${ }^{a}$, N. D. Danielson, R. E. Pauls, N. H. Mahle ${ }^{b}$, P. J. Taylor ${ }^{c}$, and L. B. Rogers

Department of Chemistry University of Georgia Athens, Georgia 30602
- NOTICE -

This report was prepared as an account of work phonsored by the United States Government. Neither the United States nor the United States Energy Research and Development Administraion, nontar their employees, nor any of their conkes any subcontractors, or their enploy assumes any legal warranty, express or ility for the accuracy, completeness liabiliy or respos information, apparatus, product or or ses disclosed, or represents that its use would not intrnge privately owned rights.

\footnotetext{
a. Present address: Department of Chemistry Purdue University

W. Lafayette, Indiana 47907

b. Present address: Dow Chemical Company Midland, Michigan 48640; work done at Purdue University

c. Present address: Department of Chemistry

Wright State University

Dayton, Ohio 45431;

work done at Purdue University
} 


\section{DISCLAIMER}

This report was prepared as an account of work sponsored by an agency of the United States Government. Neither the United States Government nor any agency Thereof, nor any of their employees, makes any warranty, express or implied, or assumes any legal liability or responsibility for the accuracy, completeness, or usefulness of any information, apparatus, product, or process disclosed, or represents that its use would not infringe privately owned rights. Reference herein to any specific commercial product, process, or service by trade name, trademark, manufacturer, or otherwise does not necessarily constitute or imply its endorsement, recommendation, or favoring by the United States Government or any agency thereof. The views and opinions of authors expressed herein do not necessarily state or reflect those of the United States Government or any agency thereof. 


\section{DISCLAIMER}

Portions of this document may be illegible in electronic image products. Images are produced from the best available original document. 


\begin{abstract}
ABST RACT
A high-precision gas chromatograph was used in conjunction with a quadrupole mass spectrometer and an on-line computer to study the fractionation of carbon and oxygen isotopes in cartion dioxide on two solid adsorbents and on a variety of liquid phases. For adsorption studies, the relative retention is reported as a function of temperature on Porapak $Q$ and silica gel. On the former, a values are on the order of $1.0027 \pm 0.0001$ (standard deviation) for the carbon isotopes and $1.0011 \pm$ 0.0002 for the oxygen species, while on silica gel, the values are $1.0031 \pm 0.0004$ and $1.0050 \pm 0.0008$, respectively. Differential thermodynamic data have been reported. Using diethanolamine and polyethylene imine columns in gas-liquid chromatography, greater fractionation. was found. However, column bleed and decomposition of the liquid phase werc serious problems.
\end{abstract}


INTRODUCTION

Concern over the hazard of radioactive isotopes has encouraged the replacement of carbon-14 by carbon-13 as a tracer atom in diagnostic and metabolic studies carried out in clinical and medical laboratories. Uses can be expected to increase rapidly because $90 \%$ enriched ${ }^{13} \mathrm{CO}_{3}$ is now available at a reasonable cost (1). 'As a result, methods for the analysis of ${ }^{13} \mathrm{C}$ should become increasingly widespread. Caprioli et al. (2) have reported that accurate and precise abundance measurements of stable isotopes can be made, both in static samples and in gas chromatographic peaks, using a quadrupole mass spectrometer. In addition, infrared measurements and ${ }^{13} \mathrm{C}$ NMR can be used to obtain structural information about complex molecules using ${ }^{13} \mathrm{C}$-labeled reactants.

Separations by gas chromatography, using either packed or open-tubular. columns, could, in principle, provide an alternate means for analyzing some mixtures of stable isotopic species. Although the emphasis in reported isotopic separations has been on deuterated molecules, there have been low-temperature separations of nitrogen, oxygen, and carbon isotopes $(3,4)$. The isotope effect in gas chromatography is small so that long columns and long retention times a re normally required for analytical separations that do not employ a mass spectrometer. In a clinical laboratory, the disadvantage of time-consuming shromatographic analyses might be offset by the relatively low cost and easy maintenance of the equipment when compared to mass spectrometry.

Chromatographic methods for isotope analysis result from the relationships between the relative vapor pressures of isotopic species and differences in rotational, vibrational, and translational frequencies of each species in the condensed phase, as worked out by Bigeleisen (5). He has pointed out that the lighter isotope does not always have the higher vapor pressure as would be expected, that those effects are temperature dependent, 
and they should be predictable from shifts in internal frequencies related to molecular structure. Cross-over temperatures, where the relative vapor pressures of isotopic species are reversed, can also be predicted. In fact, these effects have been observed by Bruner et al. (4) in adsorption studies of isotopic methanes.

Van Hook (6) has applied the theory directly to gas chromatography after dropping some of the terms in Bigeleisen's expressions. He has shown that isotopic separations can be directly related to differences in measured spectroscopic frequencies of isotopic species in the gas and the condensed phases. Spectroscopic measurements of frequency shifts due to isotope effects should be even more meaningful for adsorbed species if spectra were obtained for each species when adsorbed on the chromatographic support under conditions used to obtain the chromatographic data. The measurement may or may not be possible because of the difficulties due to decomposition of the support (laser Raman) or to spectral absorption or fluorescence by the adsorbent.

Karger (7) has suggested that differential thermodynamic data could be obtained for isotopic species by gas chromatography, but that those values would be relatively small because of the small separation factors between isotopes. One of our goals was to determine if meaningful data would be obtained rapidly on short columns by using high-precision chromatographic techniques combined with a mass spectrometer as a selective mass detector. In that way, direct measurement of relative retentions could be made. It should then be possible to correlate the chromatographic data with the spectroscopic.

The fractionation of carbon and oxygen isotopes in carbon dioxide by adsomtion on Porapak $Q$ and silica gel has been reported by Gunter and Gleason (8), but the extent of fractionation they obtained was not clear. To get those details, studies of relative retention as a function of temperature were made on short, packed columns with the intention of optimizing the conditions for separation before attempting more 
nearly complete analytical separations using long capillary columns coated with an adsorbent or a liquid phase. Differential thermodynamic properties have been determined for both the oxygen and the carbon isotopes from the relative retention values.

\section{EXPERIMENTAL}

Reagents. Diethanolamine, $97 \%$ pure (Aldrich Chemical Co.), polyethylene imine (Supelco, Inc.), 80/100 mesh Porapak Q (Waters Associates, Inc., Batch 718), 100/120 mesh silica gel (Davison Chemical Co.), and the 100/120 mesh acid-washed, HMDS-treated Chromasorb W (Alltech Associates; Inc.) were used as received. Silanization reagents included Silyl 8 and hexamethyldisilane (HMDS) from Pierce Chemical Co. The HMDS was diluted to $20 \% \mathrm{v} / \mathrm{v}$ with toluene for treating the WatsonBiemann separator.

Carbon dioxide and argon were used as received from Selox, Inc. Helium (Selox, Inc.) was purified by passing it through copper filings at $500^{\circ} \mathrm{C}$ and then Linde molecular sieve 5A (Union Carbide Corp.)

Apparatus. On-line data acquisition and analysis were performed using a PDP $11 / 20$ minicomputer system described previously $(9,10)$. Gas chromatographs were assembled in our laboratory from component parts. A Seiscor Model VIII sampling valve (Seismograph Service Co.) having a $0.5 \mu l$ internal loop and a Valco Model CV-8-HPa pneumatic valve (Valco Instruments, Inc.), having two matched $10 \mu$ l sample loops, were used. In studies below $55^{\circ} \mathrm{C}$, chromatographic columns were immersed in a 4-liter Dewar of $95 \%$ ethanol which was cooled by a Flexicool' refrigerated probe (FTS Systems, Inc.) and heated by a 300-watt metallic heater. Studies above $55^{\circ} \mathrm{C}$ were carried out in a custom-made cylindrical air bath, $20 \mathrm{~cm} \mathrm{i.d.} \mathrm{and} 23 \mathrm{~cm}$ high. A $15 \mathrm{~cm}$ squirrel. 
cage fan, turning at 1700 r.p.m., circulated air from the bottom of the oven past a 22-gauge nichrome wire heater. The temperature in each system was regulated using a Melabs Model CTC-IA proportional controller and a Model 1102 low-pass platinum sensor (Melabs, inc.). The low-temperature bath was held constant within $\pm 0.01 \mathrm{C}^{\circ}$ and the air bath within $\pm 0.03 \mathrm{Co}$. All electronics for the chromatographic system, as well as a temperature-conditioning column for the carrier gas, were thermostated at $35^{\circ} \mathrm{C} \pm 0.2^{\circ} \mathrm{C}$ to increase stability and improve precision.

The outlet of the chromatograph was connected to the ion source of a UTI $100 \mathrm{C}$ quadrupole residual gas analyser (Uthe Technology Intemational) which was capable of scanning the mass range 1 to $300 \mathrm{a} . \mathrm{m} . \mathrm{u}$. in $75 \mathrm{~ms}$. A Channeltron (Bendix Corp.) electron multiplier was used to measure ion currents. The accuracy and precision of abundance measurements of stable isotopes with this instrument have been reported in a previous study (11). The vacuum in the mass spectrometer chamber was monitored using a Varian NRC Model 386 ionization gauge. The quadrupole and chromatograph were connected through a Watson-Biemann effusion separator (12) constructed from a $5 \mathrm{~cm} \times 3.2 \mathrm{~mm}$ i.d. porous glass frit having a $1_{\mu}$ average pore size (Corning Glass Works). The separator and chromatographic column outlet were connected by a 30 $\mathrm{cm} \times 0.5 \mathrm{~mm}$ i.d. $316 \mathrm{SS}$ tube; the separator and ion source were connected by a Hoke Model 1314G4Y micrometering valve. The entire gas chromatograph-mass spectrometer interface was silanized by soaking for 2 hours in $20 \% \mathrm{v} / \mathrm{v}$ HMDS in toluene at $50^{\circ} \mathrm{C}$. After mounting, it was wrapped with heating tape.

Procedures. Carbon dioxide-argon mixtures were prepared in a 1-liter reservoir. Sample sizes were changed by selecting the $0.5 \mu$ or $10_{\mu}$ l sampling valve and by changing the relative partial pressure of each gas in the sample mixture. Carbon dioxide-to-argon pressure ratios of $1: 4,1: 1$, and $4: 1$ were used, but most experiments were done using the 4:1 ratio. 
Chromatographic columns were prepared from $2.2 \mathrm{~mm}$ i.d. 316 SS tubing (Alltech Associates, Inc.) after it had been rinsed successively with methanol, methylene chloride, and acetone. A 3.05 m Porapak $Q$ column was packed and conditioned with $5 \mathrm{ml} / \mathrm{min}$ helium at $150^{\circ} \mathrm{C}$ for 6 hours. Silica gel columns of $3.05 \mathrm{~m}$ and $1.2 \mathrm{~m}$ were prepared. The longer packed columin was silanized with $250 \mu \mathrm{l}$ of Silyl 8 at $170^{\circ} \mathrm{C}$ (injected on the column in $10 \mu$ portions at intervals of 5 minutes). Both of the silica gel columns were conditioned with $5 \mathrm{ml} / \mathrm{min}$ helium at $230^{\circ} \mathrm{C}$ for 12 hours. Diethanolamine $(10 \%$ by weight) was coated on Chromasorb $W$ then packed in a $3.05 \mathrm{~m}$ column and conditioned at $1050 \mathrm{C}$ for 2 hours using $2 \mathrm{ml} / \mathrm{min}$ helium. Polyethylene imine, $20 \%$ by weight on Chromasorb W, was conditioned in a $3.05 \mathrm{~m}$ column with $5 \mathrm{ml} / \mathrm{min}$ helium at $150^{\circ} \mathrm{C}$ for 10 minutes followed by 2 hours at $120^{\circ} \mathrm{C}$.

Chromatographic column temperatures were determined using a type $J$ thermocouple (Omega Engineering, Inc.) referenced to an ice bath at $0^{\circ} \mathrm{C}$. The emf generated by the thermocouple was recorded by the computer using a Keithley Model 160 Digital Multimeter connected to an Anscan Model 3700 analog-to-digital converter (Beckman Instruments) $(A D C)$. Helium carrier gas was reduced from tank pressure to 100 psig. and, after purification, equilibrated to $35^{\circ} \mathrm{C}$ in the thermostated chamber described above. The flow rate was controlled by a Chromatrol dual-column electronic controller (Applied Materials, Inc.). Flow rates were read both visually from the front panel of the controller and by the computer using the Beckman Anscan.

Chromatographic sampling was done under computer control using pneumatically operated valves to refill the sample loop and to inject samples.

The mass spectrometer was controlled by the computer for data acquisition. Massto-charge ratios were selected using a Model 14 QM digital-to-analog converter 
(Analog Devices, Inc.). Then selection of the electrometer sensitivity $\left(10^{-5}\right.$ to $10^{-12}$ amps) for the electron multiplier detector was made through an 8-bit input/output data latch described previously (13). The output signal was read by a Hewlett-Packard Model 2212A voltage-to-frequency converter. Individual mass chromatograms were stored on DECtape (Digital Equipment Corp.).

Data Acquisition. For each series of experiments, the region of optimal flow rate was estimated from the minimum in the Van Deemter plot at the first temperature studied. Between two and three hours were allowed for temperature equilibration before starting each experiment. Then, data were acquired under computer control using a multiple-ion-monitoring technique. In each experiment, 240 readings of intensity vs time were acquired for each isotope as the sample eluted. The rate of data acquisition varied between 1 and $4 \mathrm{~Hz}$ depending on the peak width. Standard deviations were calculated from a minimum of six replicates at each temperature. Temperatures were studied in random order for the determinations of $\Delta\left(\Delta \underline{H}^{\circ}\right)$ and $\Delta\left(\Delta \underline{S}^{\circ}\right)$. Separations of the carbon and oxygen isotopes had to be studied in separate experiments because the integration times needed to get the desired precision did not permit measurements to be made on three masses at the desired frequency.

Calculations. Peak locations were calculated by measuring their maxima. Gram polynomials (14) were used to fit 21 data points around the maximum in the mass chromatogram for each isotope. Then, the retention time, $\underline{t}_{\mathbf{r}^{\prime}}$ was computed from the elapsed time between sample introduction and calculated peak maximum. The $\underline{t}_{\underline{r}}$ for the second mass was also corrected for the time delay (a maximum of $15 \mathrm{~ms}$ ) in the reading process so as to approximate more closely the actual time at which the reading was màde.

Calculations of thermodynamic variables from chromatographic data have been reviewed in detail by Karger (7) and summarized by Culp et. al. (15). In the present 
study, the relative retention, $\alpha$, was calculated from

$$
\left.\left.a=\underline{t}_{\underline{r}_{2}}-\underline{t}_{\underline{a}}\right) / \underline{t}_{\underline{r}_{1}}-\underline{t}_{\underline{a}}\right)=\underline{K}_{\underline{2}} / \underline{K}_{\underline{1}}
$$

where ${\underline{t_{r}}}_{\underline{1}}$ and ${\underline{t_{r}}}_{\underline{r}_{2}}$ are uncorrected retention times; $\underline{t}_{\underline{a}}$ is the retention time of the inert gas, and $\underline{K}_{1}$ and $\underline{K}_{2}$ are distribution ratios. The measurement of a was made from each chromatogram so as to use the internal standard approach to gain precision instead of averaging individual values of $\underline{K}$ for each species. Differential standard molar free energies were calculated from

$$
\Delta\left(\Delta \underline{G}^{0}\right)=-\underline{R} I \ln \alpha
$$

where $\underline{R}$ is the gas constant, and $I$ is the absolute temperature. A linear least-squares analysis of $\ln \alpha$ vs $1 / T$ was used to calculate $\Delta\left(\Delta \underline{H}^{\circ}\right)$ and $\Delta(\Delta \underline{S}$ ) from

$$
\ln \alpha=-\frac{\Delta\left(\Delta \underline{H}^{\circ}\right)}{\underline{R} \underline{I}}+\frac{\Delta\left(\Delta \underline{S}^{\circ}\right)}{\underline{R}} .
$$

\section{RESU LTS}

Preliminary studies. Experiments similiar to those reported by Gunter and Gleason (8) were carried out so as to decide which chromatographic systems merited further investigation and to determine the best experimental conditions for more detailed studies. We verified the fact that measurable fractinnations could be obtained with our apparatus using $3.05 \mathrm{~m}$ columns of Porapak $Q$ and silica gel. However, measurements on Porapak $Q$ had to be made at subambient temperatures because of the small differences in retention times. Furthermore, the silica gel column had to be silanized so as to minimize tailing. The effects of changing sample size were examined on both Porapak $Q$ and silica gel, and no significant changes in the retention time and peak width at half height were found as the relative partial 
pressures of carbon dioxide and argon in the sample were changed from 1:4 to 1:1 to $4: 1$. A pressure ratio of $4: 1$ was used in subsequent experiments.

Adsorption chromatography. The quantitative effects of changes in temperature are not easily predicted for fractionations of isotopic species. Bruner et al. (4) have shown for simple molecules that there may be an inversion temperature where the relative elution of isotopic species changes. Furthermore, that temperature will not be the same for all species.

To explore those effects, fractionations of both carbon-13 and oxygen-18 were investigated on Porapak $Q$ between $-13^{\circ} \mathrm{C}$ and $-25^{\circ} \mathrm{C}$. Below $-25^{\circ} \mathrm{C}$, peak broadening was a serious problem, and above $-13^{\circ} \mathrm{C}$, fractionations were small and very difficult to measure. Retention times increased from 700 to 1100 seconds as the temperature decreased, and peak widths measured at half height increased from approximately 50 seconds to 120. Actual separations of the peak maxima were on the order of 1.5 to 3 seconds in the case of the carbon isotopes and 0.6 to 1.2 seconds for the oxygen isotopes. Changes in the relative retention, $\alpha$, and the differential standard molar free energy, $\left(\Delta\left(\Delta \underline{G}^{\circ}\right)\right)$, as the temperature decreased are presented in Table I for the ${ }^{12} \mathrm{C} /{ }^{13} \mathrm{C}$ and ${ }^{16} \mathrm{O} / 18 \mathrm{O}$ isotopic species. It is important to note that the $\Delta\left(\Delta \mathrm{G}^{\circ}\right)$ values are reported in small calories.

From the $3.05 \mathrm{~m}$ silica gel column at subambient temperatures, the carbon dioxide eluted as a very broad peak, and it was detected only as a slowly drifting baseline. Temperature studies on silica were therefore carried out at higher temperatures between $39^{\circ} \mathrm{C}$ and $50^{\circ} \mathrm{C}$ (Table I). Retention times changed from 620 to 840 seconds and peak widths measured at half height from approximately 100 to 160 seconds as the temperature decreased. Differences in the peak maxima of the carbon isotopes changed from about 1.5 to 2.5 seconds as the temperature decreased, and, for the oxygen isotopes, the change was from 2.5 to 4 seconds. 
Relative retentions in these studies were measured with standard deviations of 0.01 to $0.04 \%$ on Porapak, and 0.02 to $0.08 \%$ on silica gel. The better precision in the former being due to having narrower peaks that resulted in larger signals: Precision for the relative retention of carbon isotopes was generally better than that for oxygen. isotopes due to the larger signals from the relatively more abundant ${ }^{13} \mathrm{C}$ compared to 180 signals. The precision of those measurements approached that reported for highprecision gas chromatography using conventional detectors (15).

Additional studies were also carried out on the $1.2 \mathrm{~m}$ silica gel column at $100^{\circ} \mathrm{C}$ to more closely duplicate the conditions used by Gunter and Gleason (8). However; no separation of maxima was observed at the higher temperature.

Values for $\Delta\left(\Delta H^{\circ}\right)$ and $\Delta\left(\Delta S^{\circ}\right)$ reported in Table $\|$ were calculated from a linear least squares analysis of $\ln$ a $v s, / T$ and equation 3 . These values can only be interpreted as approximations of the differential enthalpies and entropies of adsorption for the isotope pairs on each adsorbent because of the magnitudes of the standard deviations reported for the relative retentions, compared to the changes in a with temperature. However, the trend of increased $\alpha$ at lower temperatures does appear to be real. Two t-tests, using the data at mid-range relative to data at the high and low extremes of temperature, showed that the null hypothesis could be rejected at the $80 \%$ confidence level in all but one case, where it was $60 \%$. The results, therefore, indicate that significant increases in fractionation did occur for each system as the temperature decreased over the range studied. In most cases, the uncertainty appeared in the second significant figure of the result.

Gas-liquid chromatography. The small fractionations on packed adsorption columns indicated that very long columns would be needed to achieve an isotopic separation of carbon dioxide that could be measured using a non-selective detector 
such as one based upon thermal conductivity. Because of the large pressure drop across very long packed columns, complete separation of the isotopes would only be practical using open-tubular columns. Calculations based on data obtained from columns of Porapak $Q$ and silica indicated that a $1000-2000 \mathrm{~m}$ column would be required for 0.5 resolution. Therefore, liquid phases that could be used to coat open-tubular columns were investigated using short packed columns. Because countercurrent distillation of carbon dioxide with various amines (16) has been used to prepare species enriched in ${ }^{13} \mathrm{C}$, our choice of possible liquid phases included similar compounds. However, a wide variety of common chromatographic liquid phases were also investigated so as not to miss a promising system. Liquids on which significant isotopic fractionation was not observed included $\beta \beta$-oxydipropionitrile, DC-710, silar $10 C$, silar $5 C$, Hallcomid M-18-0L, hexamethylphosphoramide, di (2-ethyl hexyl) sebacate, and polyvinylpyrrolideone,

The best fractionations for both carbon and oxygen isotopes were obtained on a diethanolamine column at $79^{\circ} \mathrm{C}$ and are reported in Table III. Retention times were on the order of 110 seconds with separations of peak maxima of about 2 to 3 seconds. Values for both the relative retention and $\Delta\left(\Delta \underline{G}^{\circ}\right)$ were considerably larger than the corresponding values obtained for adsorption, but peak widths were also greater, being 30 to 40 seconds wide at half height. Furthermore, despite the relatively high boiling point of diethanolamine, $278^{\circ} \mathrm{C}$, column bleed at $79^{\circ} \mathrm{C}$ was a very serious problem on the packed column. When the column was operated at $85-90^{\circ} \mathrm{C}$ for 5 to 6 hours and then returned to the lower temperature, a values were found to have changed from $1.043 \pm$ 0.002 to $1.033 \pm 0.001$, and retention times from 140 seconds to 110 seconds. The a values as well as the retention times at the lower temperature were found to have changed significantly from the earlier values. We believe this effect was caused by 
changes in loading due to column bleed. The change in a indicates that more than one chromatographic process must have been contributing to the fractionation. Because of the difficulties due to column bleed and the change in ' $\alpha$, studies on open-tubular columns were not attempted.

Isotope fractionation was also observed on the polyethylene imine column. The relative retention found. for the carbon isotopes was approximately 1.02 . Although this magnitude of a separation was encouraging, further study indicated that reproducible columns could not be prepared. Studies with both packed columns and open-tubular columns suggested that the retention characteristics of polyethylene imine changed with time, possibly by decomposition. For example, although the retention time for cartion dioxide as a whole did not change appreciably, the isotopic fractionation observed for a particular column decreased essentially to zero after a relatively short period of use.

\section{DISCUSSION}

On silica gel at $100^{\circ} \mathrm{C}$, Gunter and Gleason (8) reported that ${ }^{13} \mathrm{C}^{16} \mathrm{O}_{2}$ eluted first; ${ }^{17} \mathrm{C}^{16} \mathrm{O}_{3}$ second, and ${ }^{12} \mathrm{C}^{16} \mathrm{O}^{18} \mathrm{O}$ last. Under similar conditions, we did not observe any measurable fractionations. However, using longer columins and lower temperaturcs, we found thut the carbon isotopes eluted in the reported order, but that the order of the oxygen isotopes was reversed; the ${ }^{18} \mathrm{O}$ eluted before the ${ }^{16} \mathrm{O}$ and before ${ }^{13} \mathrm{C}$. However, we shall show that those discrepencies can be explained by the results of our studies of a values as a function of temperature.

In studying the separation of isotopic methanes, Bruner et al. (4) found that isotopic fractionations exhibited a temperature dependence that, in many cases, resulted in a cross-over of the elution onder of a pair of isotopes. By extrapolating 
our plot of $\log$ a vs $1 /$ f for the oxygen isotopes, an a value of unity was found to occur just below $100^{\circ} \mathrm{C}$. (This extrapolation assumes that the temperature effect was linear, which may or may not be the case.) This explains our failure to see a measurable fractionation at $100^{\circ} \mathrm{C}$ and why our elution order at lower temperatures differed from that reported by Gunter and Gleason (8). The same type of calculation was also performed on the a values for carbon. Since fractionation of carbon isotopes on silica changed much less with temperature than the oxygen isotopes, the calculated cross-over temperature was higher, near $150^{\circ} \mathrm{C}$.

For the Porapak $Q$ system at subambient temperatures, we found that ${ }^{13} \mathrm{C}^{16} \mathrm{O}_{2}$ eluted first, ${ }^{12} \mathrm{C}^{16} \mathrm{O}^{18} \mathrm{O}$ second, and ${ }^{12} \mathrm{C}^{16} \mathrm{O}$, last. Gunter and Gleason (8) reported. similar results for the caibon isotopes, but they did not report fractionation for the oxygen species. We believe we were able to measure the oxygen system because we operated at subambient temperatures where retention times were longer and separations of peak maxima were greater. Note that the elution order of the isotopes in our study was not the same on Porapak $Q$ and on silica gel. However, not only was that same order of retention of ${ }^{13} \mathrm{C}$ verified on Porapak $Q$ using a $1: 1$ mixture of ${ }^{12} \mathrm{C}^{16} \mathrm{O},:{ }^{13} \mathrm{C}^{16} \mathrm{O}$, (Mound Laboratories, Monsanto Research Corp.), but also the same value of a was found for the enriched mixture as for the natural-abundance samples.

It is important to realize that we were able to make direct meinsurements on relatively short columns: where the differences in the position of peak maxima were usually less than $1 \%$ and differences in the standard molar free energies were between 0.5 and 5.0 calories. Karger $(7)$ has suggested that measurements of $\Delta\left(\Delta G^{\circ}\right)$ on the order of 2 calories should be possible using high-efficiency open-tubular columns, and that separate chromatograms for each species might be required if peaks were badly overlapped. We avoided the latter problem by using a mass-sensitive detector which allowed us to obtain separate chromatograms for each isotopic species. Furthermore, 
Culp et al . (15) showed that a high-precision gas chromatograph could be used reliably to measure small differences in thermodynamic properties when both components were injected together so that relative retention times could be determined from the same chromatogram. By connecting the gas chromatograph to the mass spectrometer and by using an on-line computer to acquire data, we were able to measure both isotopes in the same sample and, the refore, determine a from one chromatographic run. Very careful control of chromatographic temperatures and carrier gas flow improved the precision of the measurements of $\alpha$ as a function of temperature.

Accurate retention-time measurement in gas chromatography is best done by locating the position of the peak mean. However, it is well known that significant errors can result if the peak does not return to baseline. In the studies reported here, calculations of the peak means were not used because peaks were badly tailed and often did not return to baseline within a reasonable time. Furthermore, ${ }^{18} \mathrm{O}$ and ${ }^{13} \mathrm{C}$ peaks were noisy. Instead of peak means, we used a least-squares analysis to fit 21 data points around the maximum in each chromatogram and then calculated relative retentions from the positions of the calculated peak maxima. Since we were studying isotopes in the same compound, peak shapes should have been virtually identical so the a values calculated from maxima and means should also have been insignificantly different.

Because curve fitting the top of peaks was difficult when noise was present, we also explored the possibility of determining the separations of chromatographic peaks of isotopic species by calculating the relative peak displacements on the fronts of the peaks at their hall heights. When looking at less abundant isotopes, we used greater electrometer sensitivities to make the observed heights more nearly equal. In addition, after the baseline had been subtracted, each chromatogram was then normalized to 
unit height. A linear least-squares fit of 3 points on each side of the datum nearest the half height allowed us to determine by interpolation the exact time coordinate of the half height on the front of the peak for each isotope. The difference between those coordinates for a pair of isotopes gave us a very good indication of the separation of the two peaks. As an example, for the fractionation of carbon isotopes on Porapak $Q$ at $-18^{\circ} \mathrm{C}$, the separation calculated by curve fitting the top of the peak was $1.84 \pm$ 0.12 seconds while at half-heights, the separation was $1.97 \pm 0.07$ seconds. The slightly greater values for the separation $(5-10 \%)$ and the noticeably better precision (a factor of 2) calculated from the half heights appeared to be typical of most of the cases we compared.

Studies are continuing in our laboratory to correlate spectroscopic measurements with the values of relative retention reported here. The possibility of predicting isotope fractionation by using differential shifts in infrared and Raman frequencies of isotopic species in the gas phase and of species adsorbed on chromatographic supports is being investigated. Bruner et al. (4) have suggested that differences between the observed adsorption behovior and that predicted from an equilibrium between a pure liquid and its vapor should provide information concerning isotopic interactions with the column material. Shifts in frequencies due to isotopic substitution will, therefore, have to be measured for species adsorbed on the chromatographic support under the same experimental conditions as those used for determining $\alpha$.

\section{ACKNOWLEDGEMENTS}

The authors wish to thank J. W. Amy and W. E. Baitinger for their suggestions and discussions. The assistance of David Keisel in obtaining some of the preliminary data is gratefully acknowledged. This work was supported in part by the Energy Research and Development Administration Contract No. $E(38-1)-854$. 
0

\section{REFERENCES}

1. Mound Laboratory, Monsanto Research Corp., Miamisburg, Ohio.

2. R. M. Caprioli, W. F. Fies, and M. S. Story, Anal. Chem., 46, 453A (1974).

3. G. Cartoni and M. Possanzini, J. Chromatog., 39, 99 (1969).

4. F. Bruner, G. P. Cartoni, and A. Liberti, Anal: Chem., 38, 298 (1966).

5. J. Bigeleisen, J. Chem. Phys., 34, 1485 (1961).

6. W. A. Van Hook, J. Phys. Chem., 71, 3270 (1967).

7. B. L. Karger, Anal. Chem., 39, 24A (1967).

8. B. D. Gunter and J. D. Gleason, J. Chromatog. Sci., 9, 191 (1971).

9. J. E. Davis, A. Shepard, N. Stanford, and L. B. Rogers, Anal. Chem., 46, 821 (1974).

10. T. A. Maldacker, J. E. Davis, and L. B. Rogers, Anal. Chem., 46, 637 (1974).

11. R. E. Pauls, A. T. Shepard, L. B. Rogers, and N. Mahle, "The UTI Joumal", Vol. 2, 1975.

12. J. T. Watson and K. Biemann, Anal. Chem., 37, 844 (1965).

13. J. E. Davis and E. D. Schmidlin, Chem. Instrum., 4, 169 (1973).

14. H. T. Davis, "Tables of the Mathematical Functions", Vol. II, The Principia Press, San Antonio, Texas, 1963, p. 307.

15. R. A. Culp, C. H. LochmUller, A. K. Moreland, R. S. Swingle, and L. B. Rogers, J., Chromatog. Sci.i, 9, 6 (1971).

16. J. P. Agrawal, Sep. Sci., 6, 819 (1971). 


\section{TABLE I}

RELATIVE RETENTIONS AND $\Delta\left(\Delta G^{\circ}\right)$ AS A FUNCTION OF TEMPERATURE FOR THE FRACTIONATIONS OF THE ISOTOPIC SPECIES OF CARBON DIOXIDE ON PORAPAK $Q$ AND SILICA GEL.

A. PORAPAK $Q$

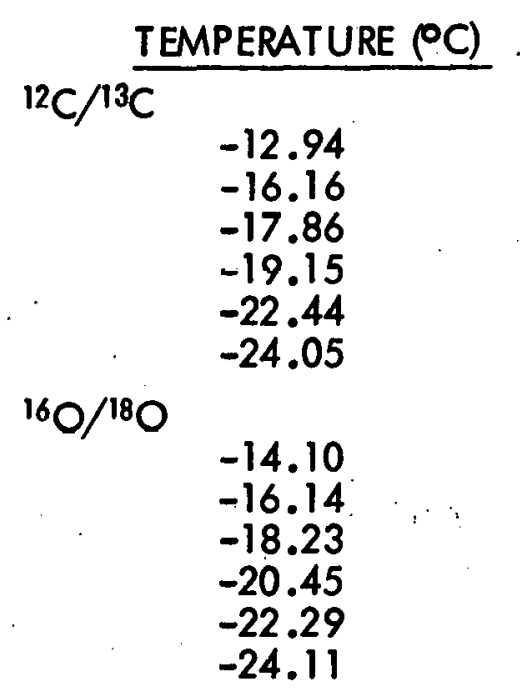

B. · SILICA GEL

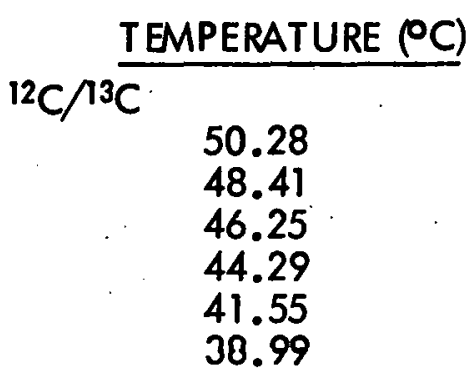

$160 / 180$

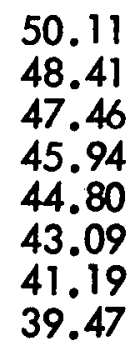

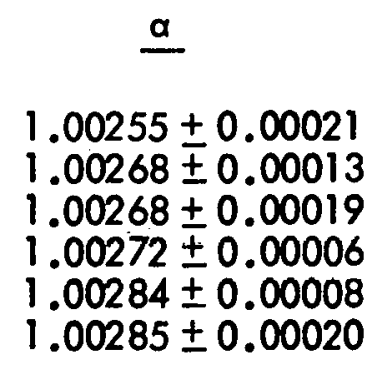

$1.00096 \pm 0.00008$

$1.00085+0.00010$

$1.00119 \pm 0.00023$

$1.00102 \pm 0.00040$

$1.00113 \pm 0.00008$

$1.00121 \pm 0.00036$
$\Delta\left(\Delta \underline{G}^{\circ}\right)$, (calories)

$-1.32 \pm 0.11$

$-1.36 \pm 0.07$

$-1.36 \pm 0.10$

$-1.37 \pm 0.03$

$-1.41 \pm 0.04$

$-1.40 \pm 0.10$

$-0.49 \pm 0.04$

$-0.43 \pm 0.05$

$-0.60 \pm 0.12$

$-0.51 \pm 0.20$

$-0.57 \pm 0.04$

$-0.60 \pm 0.18$

\section{,}

$1.00298 \pm 0.00037$

$1.00295 \pm 0.00026$

$1.00306 \pm 0.00009$

$1.00324 \pm 0.00045$

$1.00304 \pm 0.00020$

$1.00352 \pm 0.00107$

$1.00474 \pm 0.00056$

$1.00454 \pm 0.00037$

$1.00460 \pm 0.00040$

$1.00494 \pm 0.00079$

$1.00490 \pm 0.00054$

$1.00535 \pm 0.00080$

$1.00552 \pm 0.00099$

$1.00566 \pm 0.00188$
$-1.91 \pm 0.24$

$-1.89 \pm 0.16$

$-1.94 \pm 0.05$

$-2.04 \pm 0.28$

$-1.90 \pm 0.12$

$-2.18 \pm 0.66$

$-3.04 \pm 0.36$

$-2.89 \pm 0.23$

$-2.92 \pm 0.25$

$-3.12 \pm 0.50$

$-3.09 \pm 0.34$

$-3.35 \pm 0.50$

$-3.44 \pm 0.52$

$-3.51 \pm 1.16$ 


\section{TABLE \|}

$\Delta\left(\Delta H^{\circ}\right)$ and $\Delta\left(\Delta S^{\circ}\right)$ FOR THE FRACTIONATIONS OF THE ISOTOPIC SPECIES OF CARBON DIOXIDE ON PORAPAK $Q$ AND SILICA GEL.

A. PORAPAK Q

Isotopes

${ }^{12} \mathrm{C} /{ }^{33} \mathrm{C}$

$160 / 180$

B. SILICA GEL

${ }^{12} \mathrm{C} /{ }^{13} \mathrm{C}$

$160 / 180$
$\Delta\left(\Delta H^{\circ}\right)$, (calories)

$-3.48 \pm 0.05$

$-3.47 \pm 1.43$

$-8.1 \pm 4.9$

$-21.9 \pm 5.5$
$\Delta\left(\Delta \underline{\underline{S}}^{\circ}\right),($ e.u. $)$

$-0.00829 \pm 0.00001$

$-0.01160 \pm 0.00002$

$-0.0193 \pm 0.00005$

$-0.0588 \pm 0.00005$ 
TABLE III

RELATIVE RETENTIONS AND $\Delta\left(\triangle \mathrm{G}^{\circ}\right)$ VALUES FOR FRACTIONATIONS OF THE ISOTOPIC SPECIES OF CARBON DIOXIDE ON A DIETHANIOLAMINE COLUMN AT $79^{\circ} \mathrm{C}$.

Isotopes

${ }^{12} \mathrm{C} /{ }^{13} \mathrm{C}$

$160 / 180$ $\underline{a}$

$1.033 \pm 0.001$

$1.024 \pm 0.001$
$\Delta\left(\Delta \underline{G}^{\circ}\right)$, (calories)

$-22.5 \pm 0.8$

$-16.0 \pm 1.0$ 